\title{
Transitions and Trajectories for Studies of Expertise
}

\author{
by Susanne P. Lajoie
}

The transition from student to expert professional can be accelerated when a trajectory for change is plotted and made visible to learners. Trajectories or paths toward expertise are domain specific and must first be documented and then used within instructional contexts to promote knowledge transitions. This article describes how models of expertise can serve to help students attain higher levels of competence.

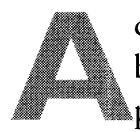

dvances made in the science of thinking and learning are based on the study of competence and developing expertise within specific curricula (Pellegrino, Chudowsky, \& Glaser, 2001). Over the years, common characteristics of expertise have been identified. ${ }^{1}$ Experts seemed to share the following characteristics: superior memory for information in their domain, better awareness of what they know and do not know, greater pattern recognition, faster and more accurate solutions (although they tend to spend more time initially analyzing problems prior to solving them), and deeper, more highly structured knowledge. Despite commonalities, a key constraint to developing expertise is that it is domain specific. Experts are experts at something, be it chess or avionics. This is important because it demonstrates that expertise is more than general intelligence.

To foster the development of expertise two goals must be achieved. The first is to determine what experts know and the second is to determine how to help novices acquire similar competencies. Identifying what experts know can help determine the trajectory towards competence for that task. This trajectory, or path, is not necessarily linear and it can have several signposts where learning transitions can take place. Once such trajectories are mapped out assessments can be designed that assess learning transitions along the road to competence. ${ }^{2}$ Research must specify how to promote transitions or changes in competence in different learning situations. Models of expertise that include different trajectories to competence can be used to design instruction and assessment for both in- and out-of-school contexts. The following two sections describe how to identify what experts know as well as how to foster the development of expertise.

\section{What Do Experts Know?}

Research on expertise has generally focused on expert-novice differences rather than the learning process (Glaser \& Bassok, 1989).

Educational Researcher, Vol. 32, No. 8, pp. 21-25
For several years, we have examined how knowledge emerges in real-world contexts, such as medicine and personal finance. Our goal, as in traditional expertise studies, is to document quantitative and qualitative differences in problem-solving strategies as a first step in improving instruction. We use cognitive methodologies, such as cognitive task analysis (CTA), to identify trajectories to competence as well as to indicate the possible transition points where instruction is needed. A major finding in our work is that there is more than one trajectory to competence. This finding must be taken into account when helping students become experts.

CTAs reveal what experts know with regard to their problemsolving plans, actions, and mental models. For example, analyses of avionics experts revealed that troubleshooters did not use one ideal solution path and solved similar problems differently (Lesgold, Lajoie, Logan, \& Eggan, 1990). However, their underlying mental models may be the same and guide their problem solving. If true, assessment must consider learning at a higher level of abstraction and a wider effective problem space in which assessment of learners takes place. CTA provides detailed maps of what experts have in common in terms of planning, strategic knowledge, actions, and interpretations as well as how they might differ from novices.

Another example of the use of CTA to study expertise in the real world was performed with surgical nurses. Just as with avionics experts, expert nurses reached similar decisions albeit by different routes, demonstrating that there are many paths to solving problems (Lajoie, Azevedo, \& Fleiszer, 1998). Differences were observed in hypothesis generation, planning of medical interventions, actions performed, results of evidence gathering, interpretation of the results, heuristics, and the overall solution paths. These characteristics lead to models of instruction where technology is used to monitor trainees and present alternative perspectives for problem solving. A computer-based learning environment, SICUN, was designed for surgical intensive care unit (SICU) nurses. In SICUN, nurses were required to post their goals before conducting patient assessments and then to specify outcomes of their assessments. For example, if their goal was to check the patient's circulatory system for adequate blood supply to the heart, they might check pulse rate as well as skin (for swelling, coloration, and other symptoms). Prior to moving on to a new goal or body system, the tutor would prompt them about the results of their assessment. Hence, plans, goals, actions, and outcomes were all built into the system with decision trees designed to encourage self-monitoring and comparison to expert problem solvers at various phases of problem solving. 


\section{Fostering Expertise Development}

Three different approaches to fostering expertise are described here: (a) conducting basic research to explicitly define transitions in expertise; (b) developing dynamic forms of assessment that lead to learning opportunities; and (c) providing explicit exemplars or models of expertise to novices. Studies of expertise inform us that becoming an expert is a transitional process. Learning in all domains is a lifelong process that can be monitored, assessed, and scaffolded. Models of expertise can assist us in determining what to monitor, how to assess, and where to scaffold learners so that they eventually become independently proficient in their chosen fields.

\section{Expertise in Transition}

While research on expertise has provided characterizations of novice and expert performance in many domains, the progression from novice to expert has not been extensively examined. Describing such a progression or trajectory toward expertise would be instructionally informative.

\section{From science to medicine}

Medical students learn from experienced practitioners and teachers (experts) and other students and residents (intermediates) during their training. Students attend lectures, participate in problem-based groups, observe others, and participate in discussions about hospital patients. To better understand developing expertise, Faremo (in preparation) examined how medical students and residents solve medical cases, compared to experts. In analyzing the cognitive processes involved in diagnostic reasoning it will be possible to represent the knowledge levels and qualitative differences in argumentation and reasoning patterns for these cohorts.

Most studies of medical expertise compare performances of experts and novices using written case problems and a cognitive approach to data analysis (Patel \& Groen, 1986). Experts and novices in these studies are separated both in place and time, and performances are denuded of realistic context. Thus, it is difficult to apply lessons from these studies to real-world medicine. On a clinical teaching ward, there are experts in many different content areas collaborating in a hierarchy to work on a given case. These experts also continuously interact within a hierarchy of novice-to-intermediate learners (medical students, interns, and residents). Wiseman (in preparation) is examining these social hierarchies and their influence on teaching and learning about diagnostic reasoning. The expert-novice chasm of past approaches can be breeched by studying distributed expertise in a collaborative setting with several patients with multiple interacting problems. Lajoie, Faremo, and Wiseman (2001) examined human medical tutorial strategies in this regard to determine how teachers and learners realistically collaborate to reveal how experts scaffold intermediate learners' processes.

\section{From expert student to expert professional}

Another example of accelerating the transformation of expert student into expert professional involves an online learning environment for undergraduates studying personal finance (Ahmad \& Lajoie, 2001). Learners were provided with access to multiple experts and distributed expertise throughout the learning envi- ronment. Experts were accessed in multiple ways. Learners could see, hear, and communicate with them through e-mail and videos. Experts provided needed feedback as students worked on assignments. An empirical analysis revealed that the embedded assessment component accounted for the most variance in contributing to transitions into expert practice and required students to reflect on their knowledge by making financial decisions based on their own profiles. Experts intervened throughout, pointing to a need for ongoing practice and refinement. Thus, the emergent aspect of expertise was reflected in the assessment artifacts.

\section{Dynamic Assessment}

One of the more successful methodologies for assessing transitions in learning is dynamic assessment. Dynamic assessment can be defined as a moment-by moment assessment of learners during problem solving so that feedback can be provided in the context of the activity (Lajoie \& Lesgold, 1992). It has been suggested that the true potential in a problem-solving situation is best assessed by supporting learners with hints or feedback to see what they may be capable of in the future without such support (Vygotsky, 1978). Dynamic assessment implies that human or computer tutors can evaluate transitions in knowledge representations and performance while learners are in the process of solving problems, rather than after they have completed a problem. Immediate feedback can then be provided to learners during problem solving, when and where they need assistance. The purpose of assessment in these situations is to improve learning in the context of problem solving.

Dynamic assessment also entails monitoring individuals' learning (Pellegrino et al., 2001). Specifically, information relevant to domain learning can be captured in a continuous record of changes in knowledge, skill, and understanding as students encounter problems of increasing complexity (Frederiksen, 1990).

The quest for dynamic forms of assessment using technology requires more research. Several methodologies are described here: (a) evidence-centered assessment (Mislevy, Steinberg, Breyer, Almond, \& Johnson, in press); (b) intelligent feedback based on student models of performance described in the Sherlock work (Lesgold, Lajoie, Bunzo, \& Eggan, 1992); and (c) overlay models that consider clusters of competency as described in the BioWorld project (Lajoie, Greer, Munsie, Wilkie, Guerrera, \& Aleong, 1995).

Evidence-centered assessment design (ECAD), provides insights for cognitive and computer scientists. Multiple forms of evidence are needed to make decisions about what individuals know in real-world settings. An ECAD approach consists of assessments linked with statistical (i.e., probability) models that can be updated as evidence is collected. ECAD enforces a rigorous methodology that starts with the claims made about students, determines what constitutes valid evidence about those claims, and determines tasks needed to gather that evidence (Mislevy et al., in press). This process ensures that assessments are based on desired proficiencies. ECAD delineates how cognitive models serve the underlying student models that guide the instruction or remediation individuals receive.

Technology can be used to assess deep-structured knowledge and emerging competence. Such patterns were documented and used to build explicit student models for the Sherlock environment, a computer-based learning environment (CBLE) designed 
to instruct avionics troubleshooting strategies (Lesgold et al., 1992). Intelligent feedback was presented dynamically as performance was assessed in context. Explicit production rules and a performance model were designed based on the analysis of explicit patterns of expert performance. Evaluating pedagogical effectiveness takes on new dimensions with CBLEs since patterns of learning indicate how skill changes over time, and provides data about transitions in learning that may not be observed by standard achievement tests (Glaser, Lesgold, \& Lajoie, 1987; Lajoie \& Lesgold 1992). The Sherlock project is an example of such gains. Avionics technicians with 20 hours of instruction on Sherlock were compared to a control group receiving on-the-job training over the same time period. The treatment group performed significantly better than the control group and equivalent to experienced technicians having several years of on-the-job experience. The average gain score for the group using Sherlock was equivalent to almost 4 years of experience (Nichols, Pokorny, Jones, Gott, \& Alley, in preparation).

Advances in cognitive science stretch the expectations for knowledge and skills we want students to develop, along with observations needed to evidence them (Mislevy et al., in press). BioWorld (a CBLE) serves as a platform for evoking evidence of knowledge and capturing complex performance as high-school students develop scientific reasoning (Lajoie, Lavigne, Guerrera, $\&$ Munsie, 2001). Students learn about diseases associated with the body systems they are studying. Patient cases are presented in the context of hospital simulations and students work collaboratively to collect evidence to confirm or refute hypotheses. Patient cases were developed with the help of experts, and a CTA established robust models of such ill-structured problems.

In BioWorld, transitions in students' learning were recorded both within and across cases. Again, by identifying what experts know, it is possible to develop assessment genres for examining transitions in learning. Expert problem-solving traces were monitored and collected dynamically, as were verbal protocols. These data determined the unique profiles of experts in terms of plans, strategies, and actions within BioWorld. Currently, different clusters of competencies were identified as experts formed a diagnostic hypothesis that served to establish benchmarks of performance within specific BioWorld learning situations (Lajoie et al., 1995). As experts solve patient cases they select relevant evidence from the patient medical history, collect pertinent information in an online medical library, perform diagnostic tests pertinent to the hypothesis, use systematic plans and actions throughout problem solving, and make final arguments based on evidence.

Once competency clusters were established, novice students were monitored by the computer in terms of the proportion of overlap of their actions with each predetermined cluster. Instructional feedback was based on the dynamic assessment of actions throughout problem solving. With practice, students became more systematic in their reasoning, and more strategic about evidence collection and hypothesis testing, indicating that instruction based on dynamic assessment can lead to transitions in learning. The aforementioned examples have demonstrated that understanding experts' knowledge can lead to better instruction through dynamic assessment. Transitions in knowledge can be monitored through these new assessment forms. Extending the use of BioWorld to higher education classrooms can complete the picture of emerging expertise in this domain. Alexander (2003) states that studies of expertise have been criticized for only looking at the extremes of the expert/novice continuum rather than the intermediate stages. In response to such criticism, we are investigating learning with BioWorld over a broader learner population. More specifically, we are examining the cognitive processes of learners from high school to medical school via BioWorld to derive a more robust model of emerging competence in diagnostic reasoning.

\section{Exemplars as Trajectories to Competence}

Expertise can be fostered by making the expertise trajectory visible to learners through models of expertise, feedback, or examples that promote the active transfer of knowledge and self-monitoring. Assessing an individual's preparedness for learning may provide the first step toward operationalizing a trajectory toward expertise (Bransford \& Schwartz, 1999). Students are better prepared to compare and contrast their response with those of experts when asked to generate an answer rather than memorize facts (Schwartz $\&$ Moore, 1998). Such self-assessments can become part of the instructional process (White, Shimoda, \& Frederiksen, 2000) whereby students develop and critique their own and others' knowledge structures. Students must adapt to new situations by transferring what they have learned in school to new situations and by adapting or restructuring learning to facilitate skill acquisition.

As mentioned, an increased focus on expertise at the intermediate level is needed, particularly in the context of schooling. Hence, models of peer performance on statistics projects that vary in levels of expertise are provided (Lajoie, Lavigne, Munsie, \& Wilkie, 1998). Expert models should be relative to what is expected of middle-school students and not to statistics experts per se. A multimedia library of exemplars provided learners with assessment criteria for statistical investigations along with performance standards. Students were provided with exemplars of what a research question entailed, an example of data collection, analysis, and interpretation. For each criterion, two levels of performance, appropriate and more appropriate, were presented for comparison purposes. The appropriate level demonstrated performance that minimally met a criterion (i.e., intermediate), whereas the more appropriate example demonstrated performance that completely met a criterion (i.e., expert).

In an attempt to provide middle-school students with the experience of being a practicing statistician or scientist, Lavigne (2000) created an environment that allows students to produce their own statistical investigations and evaluate other investigations. This dual process of producing and critiquing reflects activities that scientists engage in on a regular basis. To further extend this professional-like experience, students receive feedback on their evaluations by being presented with a researcher's critique of the projects. This approach illustrates how assessment can help students attain their potential. Emerging competence can be assessed and supported through the exemplars that provide models against which to compare their performance.

\section{Implications}

The road to competence can be shortened if systematic studies of expertise lead to improvements in instruction and assessment. 
Cognitive methodologies, such as CTA, can lead to the identifcation of expertise trajectories as well as possible transition points where instruction is needed. Trajectories toward expertise are domain specific and must be documented and then used within instructional contexts to promote knowledge transitions. Expertise studies contribute to the identification of competency models available to novices and intermediates making the transition to deeper and more integrated knowledge structures. The transition from student to expert professional can be accelerated when a trajectory for change is plotted and made visible to learners. As indicated in the avionics tutor, Sherlock, assessment can reduce the time it takes to become competent if coupled with effective feedback and practice opportunities for learner engagement in realistic contexts.

Three different approaches to fostering expertise were described here, ranging from basic research to instructional approaches that either included dynamic forms of assessment that lead to learning opportunities or presented explicit exemplars of expertise.

Basic research demonstrates that competence and expertise are domain specific. Hence the context for the study of expertise plays a role in learning and transfer. Technology can serve to integrate instruction and assessment through embedding assessment in learning contexts. However, teachers can accomplish this by using formative assessments, using student results to inform subsequent teaching efforts. The nature of the feedback must also be contextualized within the problem-solving task in order to facilitate learning and transfer.

The most important lesson of expertise research, especially in real-world domains, is that there are multiple solution paths, and clusters of processes to achieve a solution. Assessments of emerging competence must consider multiple forms of evidence so that correct solutions are not excluded based on preconceived ideas of correctness. Mislevy et al. (in press) leverage the use of technology to assemble effective models of competence in transition by pairing these with statistical models dynamically assembled in the context of problem solving. Such assessments have potential in representing knowledge and predicting what students know based on observations of behavior (Martin \& Van Lehn, 1995). In a perfect world, cognitive scientists, psychometricians and computer scientists could work collaboratively to build these complex models. Distributing expertise could enhance the ability to understand the road to competence. Although such interdisciplinary work is laborious it can lead to shareable or reusable fragments of instruction that can lead to shareable versions of expert schemas that ultimately result in better learning. Assessments intimately connected with learning contexts may ultimately have a larger payoff than large-scale assessment.

\section{NOTES}

Funding was provided by the following granting agencies: the Canadian Social Sciences and Humanities Research Council; the Quebec Ministry of Industry, Commerce, Science and Technology; Valorisation Recherche Quebec; and Office of Educational Research and Improvement, National Center for Research in Mathematical Sciences Education (NCRMSE). Many graduate students (former and current) have contributed to the work that is reported here. Special thanks go to Arshad Ahmad, Roger Azevedo, Gloria Berdugo, Janet Blatter, Andrew Chiarella, Lucy Cumyn,
Sonia Faremo, Claudia Guerrera, Genevieve Gauthier, Nancy Lavigne, Susan Lu, Carlos Nakamura, Thomas Patrick, and Jeffrey Wiseman.

'Much of this research is summarized in the now classic text by Chi, Glaser, and Farr (1988).

${ }^{2}$ Glaser (2002) coined the phrase "road to competence."

\section{REFERENCES}

Ahmad, A., \& Lajoie, S. P. (2001). The integrated learning model. In J. D. Moore, C. Redfield, \& L. W. Johnson (Eds.), Artificial intelligence in education (pp. 354-364). Amsterdam: IOS Press.

Alexander, P. A. (2003). The development of expertise: The journey from acclimation to proficiency. Educational Researcher, 32(8), 10-14.

Bransford, J. D., \& Schwartz, D. L. (1999). Rethinking transfer: A simple proposal with multiple implications. Review of Research in Education, 24, 61-100.

Chi, M. T. H., Glaser, R., \& Farr, M. J. (1988). The nature of expertise (pp. ix-xvii). Hillsdale, NJ: Erlbaum.

Faremo, S. (in preparation). Examining medical problem solving in a computer-based learning environment. Unpublished doctoral dissertation, McGill University, Montreal, Quebec, Canada.

Frederiksen, N. (1990). Introduction. In N. Frederiksen, R. Glaser, A. Lesgold, \& M. Shafto (Eds.), Diagnostic monitoring of skill and knowledge acquisition (pp. ix-xvii). Hillsdale, NJ: Erlbaum.

Glaser, R. (2002, June). The road to competence. Commencement speech delivered at McGill University, Montreal, Quebec, Canada.

Glaser, R., \& Bassok M. (1989). Learning theory and the study of instruction. Annual Review of Psychology, 40, 631-666.

Glaser, R., Lesgold, A., \& Lajoie, S. P. (1987). Toward a cognitive theory for the measurement of achievement. In R. Ronning, J. Glover, J. C. Conoley, \& J. C. Witt (Eds.), The influence of cognitive psychology on testing, Vol. 3 (pp. 41-85). Hillsdale, NJ: Erlbaum.

Lajoie, S. P., Azevedo, R., \& Fleiszer, D. (1998). Cognitive tools for assessment and learning in a high information flow environment. Journal of Educational Computing Research, 18(3), 205-235.

Lajoie, S. P., Faremo, S., \& Wiseman, J. (2001). Tutoring strategies for effective instruction in internal medicine. International Journal of $A r-$ tificial Intelligence and Education, 12(3), 293-309.

Lajoie, S. P., Greer, J. E., Munsie, S. D., Wilkie, T. V., Guerrera. C., \& Aleong, P. (1995). Establishing an argumentation environment to foster scientific reasoning with Bio-World. In D. Jonassen \& G. McCalla, (Eds.), Proceedings of the International Conference on Computers in Education (pp. 89-96). Charlottesville VA: AACE.

Lajoie, S. P., Lavigne, N. C., Guerrera, C., \& Munsie, S. (2001). Constructing knowledge in the context of BioWorld. Instructional Science, 29(2), 155-186.

Lajoie, S. P., Lavigne, N. C., Munsie, S. D., \& Wilkie, T. V. (1998). Monitoring student progress in statistics. In S. P. Lajoie (Ed.), Reflections on statistics (pp. 199-231). Mahwah, NJ: Erlbaum.

Lajoie, S. P., \& Lesgold, A. (1992). Dynamic assessment of proficiency for solving procedural knowledge tasks. Educational Psychologist, 27(3), 365-384.

Lavigne, N. (2000). Project-based investigations for producing and critiquing statistics. Unpublished doctoral dissertation, McGill University, Montreal, Quebec, Canada.

Lesgold, A., Lajoie, S. P., Bunzo, M., \& Eggan, G. (1992). SHERLOCK: A coached practice environment for an electronics troubleshooting job. In J. H. Larkin \& R. W. Chabay (Eds.), Computer assisted instruction and intelligent tutoring systems: Shared goals and complementary approaches (pp. 201-238). Hillsdale, NJ: Erlbaum.

Lesgold, A., Lajoie, S. P., Logan, D., \& Eggan, G. M. (1990). Cognitive task analysis approaches to testing. In N. Frederiksen, R. Glaser, 
A. Lesgold, \& M. Shafto (Eds.), Diagnostic monitoring of skill and knowledge acquisition (pp. 325-350). Hillsdale, NJ: Erlbaum.

Martin, J., \& Van Lehn, K. (1995) Student assessment using Bayesian nets. International Journal Human-Computer Studies, 42, 575-591.

Mislevy, R. J., Steinberg, L. S., Breyer, F. J., Almond, R. G., \& Johnson, L. (in press). Making sense of data from complex assessment. Applied Measurement in Education.

Nichols, P., Pokorny, R., Jones, G., Gott, S. P., \& Alley, W. E. (in preparation). Evaluation of an avionics troubleshooting tutoring system. Technical Report, Armstrong Laboratory, Human Resources Directorate, Brooks AFB, TX.

Patel, V. L., \& Groen, G. J. (1986). Knowledge-based solution strategies in medical reasoning. Cognitive Science, 10, 91-116.

Pellegrino, J., Chudowsky, N., \& Glaser, R. (2001). Knowing what students know. Washington, DC: National Academy Press.

Schwartz, D. L., \& Moore, J. L. (1998). The role of mathematics in explaining the material world. Cognitive Science, 22, 471-516.

Vygotsky, L. S. (1978). Mind in society. Cambridge, MA: Harvard University Press.
White, B., Shimoda, T., \& Frederiksen, J. (2000). Constructing a theory of mind and society. In S. P. Lajoie (Ed.), Computers as cognitive tools (Vol. 2) (pp. 247-271). Mahwah, NJ: Erlbaum.

Wiseman, J. (in preparation). Tutoring strategies for effective instruction in internal medicine. Unpublished Master's Thesis, McGill University, Montreal, Quebec, Canada.

\section{AUTHOR}

SUSANNE P. LAJOIE, 3700 McTavish Street, Faculty of Education, McGill University, Montreal, Quebec, Canada H3A 1Y2; susanne. lajoie@mcgill.ca. Her research interests include the design and evaluation of theory-driven computer-based learning environments for classroom and real-world applications. She has developed systems in statistics, science, and medicine and is most interested in how students reason and learn from adaptive forms of instruction.

Manuscript received January 14, 2003

Final revision received May 27, 2003

Accepted June 3, 2003

\section{Two New AERA SIGs}

AERA is pleased to announce the creation of a SIG: Comprehensive School Reform (CSR). CSR is grounded on the idea that there is a systematic process to help schools improve. CSR is an increasingly important field of study to education stakeholders because it provides a framework for schoolwide improvement that requires external support in specific areas during the implementation phase. CSR will provide a forum for an exchange of ideas and information, and encourage further research and evaluation on theories and practices related specifically to comprehensive school reform. This SIG welcomes all researchers interested in comprehensive school reform. If you are interested in joining, please contact AERA. Annual membership dues are $\$ 5$.

AERA is also pleased to announce the creation of a SIG on Educational Change. The challenges of initiating, implementing, and sustaining change are clearly multidimensional and not confined to one discipline. Consequently, this SIG adopts an inclusive, interdisciplinary, and international approach. Areas of research include large-scale reform, school-initiated change, school improvement, and classroom-level change. The SIG also addresses the impact of significant change forces on education, such as changing school demographics, accountability influences, changes in images of leadership, and the influence of market forces. Contributions from a range of disciplines and methodological approaches are welcomed. The interests of multiple audiences will be addressed, especially researchers, policymakers, and practitioners. Our AERA session will feature a keynote address by Professor Michael Fullan on the foundations, development, and new directions in the theory and practice of educational change. For more information, please contact Andy Hargreaves at stahlna@bc.edu. To join, send your information, the SIG's name, and \$5 to AERA. 